Provided for non-commercial research and education use. Not for reproduction, distribution or commercial use.

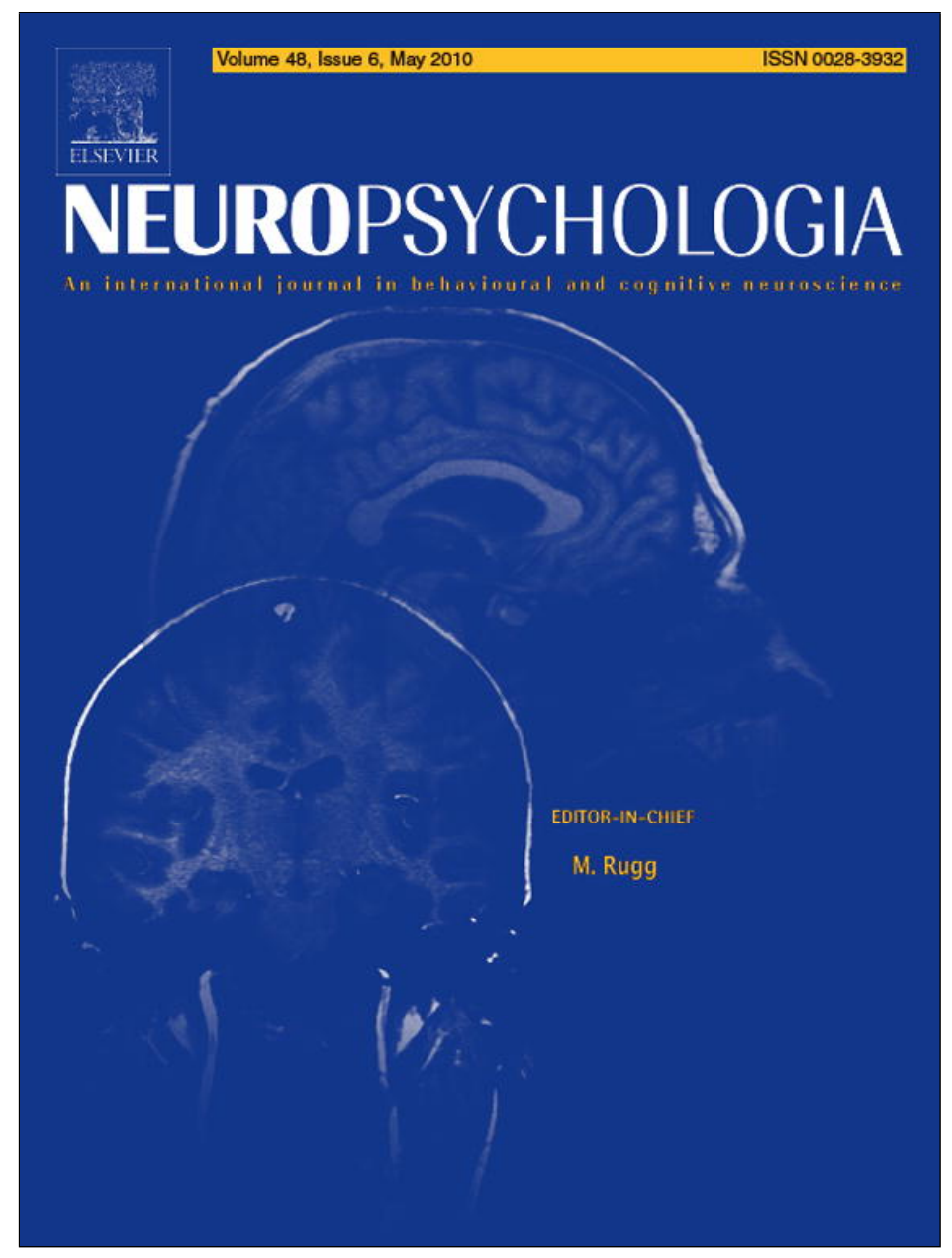

This article appeared in a journal published by Elsevier. The attached copy is furnished to the author for internal non-commercial research and education use, including for instruction at the authors institution and sharing with colleagues.

Other uses, including reproduction and distribution, or selling or licensing copies, or posting to personal, institutional or third party websites are prohibited.

In most cases authors are permitted to post their version of the article (e.g. in Word or Tex form) to their personal website or institutional repository. Authors requiring further information regarding Elsevier's archiving and manuscript policies are encouraged to visit:

http://www.elsevier.com/copyright 


\title{
The inferior, anterior temporal lobes and semantic memory clarified: Novel evidence from distortion-corrected fMRI
}

\author{
M. Visser ${ }^{a}$, K.V. Embleton ${ }^{\mathrm{a}, \mathrm{b}}$, E. Jefferies ${ }^{\mathrm{c}}$, G.J. Parker ${ }^{\mathrm{b}}$, M.A. Lambon Ralph ${ }^{\mathrm{a}, *}$ \\ a Neuroscience and Aphasia Research Unit (NARU), Zochonis Building, School of Psychological Sciences, University of Manchester, Oxford Road, Manchester, M13 9PL, UK \\ ${ }^{\mathrm{b}}$ Imaging Science and Biomedical Engineering, University of Manchester, UK \\ c Department of Psychology, University of York, UK
}

\section{A R T I C L E I N F O}

\section{Article history:}

Received 14 September 2009

Received in revised form 19 January 2010

Accepted 12 February 2010

Available online 20 February 2010

\section{Keywords:}

Semantic cognition

Anterior temporal lobes

fMRI

Semantic dementia

\begin{abstract}
A B S T R A C T
The neural basis of semantic memory generates considerable debate. Semantic dementia results from bilateral anterior temporal lobe (ATL) atrophy and gives rise to a highly specific impairment of semantic memory, suggesting that this region is a critical neural substrate for semantic processing. Recent rTMS experiments with neurologically-intact participants also indicate that the ATL are a necessary substrate for semantic memory. Exactly which regions within the ATL are important for semantic memory are difficult to detect from these methods (because the damage in SD covers a large part of the ATL). Functional neuroimaging might provide important clues about which specific areas exhibit activation that correlates with normal semantic performance. Neuroimaging studies, however, have not consistently found anterior temporal lobe activation in semantic tasks. A recent meta-analysis indicates that this inconsistency may be due to a collection of technical limitations associated with previous studies, including a reduced fieldof-view and magnetic susceptibility artefacts associated with standard gradient echo fMRI. We conducted an fMRI study of semantic memory using a combination of techniques which improve sensitivity to ATL activations whilst preserving whole-brain coverage. As expected from SD patients and ATL rTMS experiments, this method revealed bilateral temporal activation extending from the inferior temporal lobe along the fusiform gyrus to the anterior temporal regions, bilaterally. We suggest that the inferior, anterior temporal lobe region makes a crucial contribution to semantic cognition and utilising this version of fMRI will enable further research on the semantic role of the ATL.
\end{abstract}

(c) 2010 Elsevier Ltd. All rights reserved.

\section{Introduction}

The importance of the anterior temporal lobes (ATL) in semantic memory (our font of meaning for verbal and nonverbal stimuli and experiences) has become a major topic of reviews and debates in the recent neuroscience literature (Hickok \& Poeppel, 2007; Martin, 2007; Patterson, Nestor, \& Rogers, 2007). On the one hand, neuropsychological studies indicate that the ATL makes a critically important contribution in semantic cognition (Damasio \& Damasio, 1994; Lambon Ralph, Lowe, \& Rogers, 2007; Lambon Ralph \& Patterson, 2008; Patterson et al., 2007) and this has been reinforced by recent repetitive transcranial magnetic stimulation (rTMS) investigations of this same region in normal participants (Lambon Ralph, Pobric, \& Jefferies, 2009; Pobric, Jefferies, \& Lambon Ralph, 2007; Pobric, Jefferies, \& Lambon Ralph, in press). On the other hand, the fMRI literature has been relatively quiet on the topic of the ATL and semantic memory. Despite a large number

\footnotetext{
* Corresponding author. Tel.: +44 0161275 2551; fax: +44 01612752873

E-mail address: matt.lambon-ralph@manchester.ac.uk (M.A.L. Ralph).
}

of fMRI studies of comprehension, semantic memory and other aspects of semantic cognition, very few of them have implicated a relationship between the ATL and semantic memory (Patterson et al., 2007; Visser, Visser, Jefferies, \& Lambon Ralph, 2010; Wise, 2003). Instead, fMRI studies of semantic tasks have focussed on activations that arise consistently in prefrontal and posterior temporal regions (Demb et al., 1995; Devlin et al., 2000; Thompson-Schill, D'Esposito, Aguirre, \& Farah, 1997). The limited and inconsistent fMRI evidence for ATL involvement in semantic processing runs counter to the expectations arising from SD and rTMS studies and consequently, the two literatures look incompatible. In the remainder of this Section 1 we briefly review the evidence in favour of ATL involvement in semantic processing, potential limitations of fMRI in revealing ATL activation and the need for a new form of fMRI, in particular, for answering important neuroscience questions with regard to semantic cognition.

Evidence for ATL involvement in semantic cognition comes from four sources: neuropsychology, rTMS and PET- and MEG-based neuroimaging studies. Semantic impairments arise in a number of different acute and neurodegenerative neurological conditions. Of these semantic dementia (SD: Hodges, Patterson, Oxbury, \& 
Funnell, 1992; Snowden, Griffiths, Neary, \& Mann, 1989) is the best neurological model of semantic impairment because of two characteristics unique to the condition: the semantic deficit is both selective and progressive (Lambon Ralph \& Patterson, 2008; Patterson et al., 2007). The semantic impairment is selective in the sense that other aspects of memory, cognition and perception are relatively well preserved right up to and sometimes including the late stages of the disorder, allowing the inexorable degradation of concepts to be tracked over time (Lambon Ralph \& Patterson, 2008). This specificity of semantic deficit means that test performance is not clouded or confounded by other cognitive and language impairments - as they can be in other neurological conditions such as Alzheimer's disease, herpes simplex virus encephalitis, stroke aphasia and head injury. It should be noted, however, that semantic impairment in HSVE, head injury and Alzheimer's disease is also associated with damage to the inferior and lateral anterior temporal region (Hodges \& Patterson, 1995; Lambon Ralph et al., 2007; Noppeney et al., 2007) - reinforcing the conclusion from SD itself.

A debate in the literature arises when this specific semantic impairment is related to the pattern of brain damage in semantic dementia. There is no doubt that the atrophy and associated hypometabolism of SD is focused upon the anterior, inferior aspects of the temporal lobes bilaterally with consistent and substantial grey matter loss in the polar and perirhinal cortices, and the anterior fusiform gyri, bilaterally (Davies, Graham, Xuereb, Williams, \& Hodges, 2004; Nestor, Fryer, \& Hodges, 2006). Thus, the simplest and most obvious hypothesis is that these regions are critical for semantic memory (Lambon Ralph \& Patterson, 2008; Patterson et al., 2007). Given that SD is a neurodegenerative condition, there is no absolute boundary to the damage. There is, therefore, always the possibility that sub-threshold damage or dysfunction due to invading pathology occurs elsewhere and it is this more subtle, widespread damage that is the root of the patients' semantic impairment (Brambati et al., 2009; Martin, 2007). At worst, the maximal area of damage in SD is like a magician's pledge or misdirection - as neuropsychologists, our attention and subsequent theorising is erroneously drawn to the anterior temporal lobes when, in reality, semantic memory is realised elsewhere (the magician's prestige).

Convergent evidence for the role of ATL regions in semantic cognition comes from other neuroimaging and rTMS studies of normal participants. When asked to perform receptive or expressive semantic tasks, then ATL activations can be observed with PET (Mummery et al., 2000; Tranel, Grabowski, Lyon, \& Damasio, 2005; Vandenberghe, Price, Wise, Josephs, \& Frackowiak, 1996) and MEG (e.g., Marinkovic et al., 2003). Likewise rTMS to the lateral ATL, slows receptive (synonym judgement) and expressive (picture naming) tasks but has no effect on non-semantic (number-based) tests matched for overall difficulty (Pobric et al., 2007). This pattern holds whether left or right temporal poles are stimulated (Lambon Ralph et al., 2009). In contrast, the fMRI results are very inconsistent; although some studies find ATL activation during semantic processing (for review see, Binder, Desai, Graves, \& Conant, 2009; Visser et al., 2010), activation is not found in many studies using similar receptive and expressive tasks (Binder et al., 2009; Canessa et al., 2007; Chao \& Martin, 2000; Mechelli, Sartori, Orlandi, \& Price, 2006; Pilgrim, Fadili, Fletcher, \& Tyler, 2002). In the context of this convergent evidence from SD patients, PET, MEG and rTMS, the absence of ATL activation in many fMRI-based studies of semantic processing is a key and prominent divergent element in the neuroscience literature.

As noted above, a brief cursory glance at the semantic neuroimaging literature would not reveal consistent and overwhelming evidence for the involvement of the ATL in semantic memory (unlike prefrontal cortex, posterior MTG, etc.). There are strong indications, however, that this is an absence of evidence rather than evidence of absence: a recent meta-analysis of the semantic neuroimaging literature demonstrated that the likelihood of reporting ATL activation is subject to three technical issues (Visser et al., 2010). These included the imaging modality (PET is more likely than fMRI to reveal ATL semantic activations - most likely reflecting the inherent distortions found in fMRI), the field of view (many PET and fMRI studies previously used a restricted field of view $(<15 \mathrm{~cm})$ and in doing so the inferior and anterior aspects of the temporal lobes are most likely to be sacrificed) and the "control" task (ATL activation is less likely when a "rest" or low-level baseline is used - presumably because in such situations participants are able to engage in self-generated inner speech: Binder et al., 1999; McKiernan, D’Angelo, Kaufman, \& Binder, 2006). Given the great influence of fMRI research in neuroscience theorising, the technical limitations of this neuroimaging modality are critically important to understand and overcome. The ability of fMRI to detect activation is not constant across the brain. The ATL and adjacent orbitofrontal cortex reside near air-bone interfaces that cause inhomogeneities in the magnetic field leading to image distortion and signal loss when using conventional gradient-EPI (Schmithorst, Dardzinski, \& Holland, 2001; Weiskopf, Hutton, Josephs, \& Deichmann, 2006). The impact of the signal distortion on the ability to detect ATL activation was demonstrated by Devlin et al. (2000). Using a semantic categorisation task, Devlin et al. directly compared 3T GE EPI fMRI with $\mathrm{H}_{2} \mathrm{O}^{15}$ PET. Both techniques detected greater activation in prefrontal regions for the semantic over the control task, however, ATL activation (particularly in the left anterior, inferior temporal lobe) was only identified with $\mathrm{H}_{2} \mathrm{O}^{15}$ PET (see Fig. 2a, reproduced with permission).

Functional neuroimaging of the ATL will be critical in answering the next cycle of research questions about the neural basis of semantic cognition. These include which specific regions within ATL support semantic memory and whether there are divisions of labour across these regions for different types of semantic information. These questions are hard to answer with studies of SD patients because the atrophy associated with this disease covers the whole region. Repetitive TMS studies can sequentially probe different ATL regions only if they sit on the lateral surface, as the ventral surfaces of the brain are relatively inaccessible with this technique. As noted above, PET functional neuroimaging provides one method for investigating different ATL subregions but it does have a number of drawbacks in comparison to fMRI. These include: the limited number of scanning runs (typically 12 , in order to limit radiation exposure) that heavily constrain the behavioural designs that can be adopted and reduce the sensitivity of the technique; only block designs can be used; and participants can only be scanned once.

In this fMRI-based study, our aims were to confirm the hypothesis that the ATL (in addition to other regions) are implicated in semantic cognition and to use the advantages of this neuroimaging technique to begin to answer more specific neuroanatomical questions. These include: (a) is semantic memory supported by regions in the ATL bilaterally (as suggested by the bilateral atrophy of SD); and (b) is the anterior, inferior temporal region especially important (a region that is particularly vulnerable to the magnetic inhomogeneities and has the highest rate of grey matter loss in SD: Davies et al., 2004; Devlin et al., 2000)? The study also represents the first application of a novel distortion-corrected, spin echo (SE) EPI technique (based on dual-direction $k$ space transformation: Embleton, Haroon, Lambon Ralph, Morris, \& Parker, in press) that significantly reduces the problems associated with imaging the anterior and inferior temporal lobes. Although SE EPI is less sensitive to haemodynamic activation, it is not subject to the signal loss due to intravoxel dephasing that occurs with gradient echo (GE) EPI (Jones, Brookes, \& Moonen, 2001; Li et al., 2007). Substantial spatial mismapping of the signal in the phase encode direction occurs in areas of altered magnetic susceptibility in GE and SE EPI. However, 
as the total signal is preserved in SE EPI, it is possibility to apply post-processing, spatial correction that cannot be applied to GE EPI (Embleton et al., in press).

To provide an initial formal starting point in the use of an improved fMRI recipe, we deliberately conducted a comparative study by adopted the same behavioural task (rapid semantic categorisation) as used by Devlin et al. (2000). To improve the comparability we also ran a similar number of participants and imaging analysis. Devlin's behavioural task involves rapid semantic categorisation of basic level concepts and so provides an important theoretical test for different theories of ATL semantic function. Work arising from semantic dementia and rTMS suggests that the ATL supports an amodal representational hub that codes all types of concept (Lambon Ralph \& Patterson, 2008; Pobric et al., 2007; Pobric et al., in press; Rogers et al., 2004). In contrast, other work has indicated that there may be graded representational specificity along the temporal lobe such that the ATL codes for specific entities or subordinate exemplars (Martin, 2007; Martin, Wiggs, Ungerleider, \& Haxby, 1996; Tranel, Damasio, \& Damasio, 1997; Tyler et al., 2004). If the latter theories are correct then, even with successful distortion correction, no ATL activation should be found if the task requires processing of basic level concepts.

\section{Methods}

\subsection{Experimental procedure}

Eleven participants (right-handed, mean age $=32, \mathrm{SD}=6.8,5$ males) completed semantic and letter (control)categorisation tasks as described by Devlin et al (2000). The experiment comprised eight semantic blocks and eight control blocks, which lasted for $32 \mathrm{~s}$ each. The task was an ABCX categorisation task, in which participants saw three words sequentially from a single semantic category and then had to decide if a fourth word was also drawn from that category (e.g., taxi, boat, bicycle followed by AEROPLANE vs. taxi, boat, bicycle followed by SPOON). All concepts and categories were drawn from the manmade domain. Each word was presented in lowercase letters for $200 \mathrm{~ms}$, with an inter-stimulus interval of $400 \mathrm{~ms}$. The fourth word was presented in upper case and underlined to indicate that a decision was required. The trial finished with a blank screen for 2000 ms during which the participants made their decision. Semantic categorisation was contrasted with a control letter categorisation task, in which subjects viewed a series of lower-case, letter strings matched to the length of the words. Participants saw three strings of a specific letter sequentially and then had to decide if a fourth letter string (in capitals) was the same letter (e.g., rrrr, rrr, rrrrr followed by RRR or DDDD). The timings followed those used in the semantic task.

\subsection{Image acquisition}

All imaging was performed on a 3T Philips Achieva scanner using an eight element SENSE head coil with a sense factor of 2.5. The SE EPI fMRI sequence included 30 slices with TE $=75 \mathrm{~ms}$, TR $=3200 \mathrm{~ms}, 112 \times 112$ acquisition matrix, reconstructed resolution $1.875 \mathrm{~mm} \times 1.875 \mathrm{~mm}$, and slice thickness $4.2 \mathrm{~mm}$. Brief (10 volumes for each $k$ space traversal) dual direction $k$ space traversal SE EPI scans with matching parameters were made before the functional acquisitions in order to achieve sets of images matching the functional time-series but with opposing direction distortions. These pre-scans were made on each participant and were used to compute a spatial remapping matrix that could be applied to the functional time-series. The main fMRI image sequences of 160 time points were acquired with a single direction $k$ space traversal. A high resolution T2 weighted turbo spin echo scan with in-plane resolution of $0.94 \mathrm{~mm}$ and slice thickness $2.1 \mathrm{~mm}$ was also obtained as a structural reference to provide a qualitative indication of distortion correction accuracy.

\subsection{Distortion correction}

Single-shot gradient echo EPI (GE EPI) is a fast magnetic resonance imaging (MRI) method with a high signal-to-noise ratio. However, the detected MRI signal is vulnerable to geometric distortion and signal loss, especially in regions subject to large magnetic susceptibility variation (brain areas near bone or air). Moreover, this effect is increased at higher magnetic fields (Deichmann, Josephs, Hutton, Corfield, \& Turner, 2002; Jezzard, Matthews, \& Smith, 2001; Schmithorst et al., 2001; Weiskopf et al., 2006). In addition, spatial distortion is more pronounced in the phase-encoding direction than in the frequency encoding direction (Morgan, Bowtell, McIntyre, \& Worthington, 2004). Spin echo EPI (SE EPI) was used to acquire the data because this imaging technique only suffers from spatial distortion without signal loss and this licenses a post-acquisition correction procedure. Although SE EPI has lower signal-to-noise than GE EPI in unaffected areas (Jezzard et al., 2001), it has signifi-

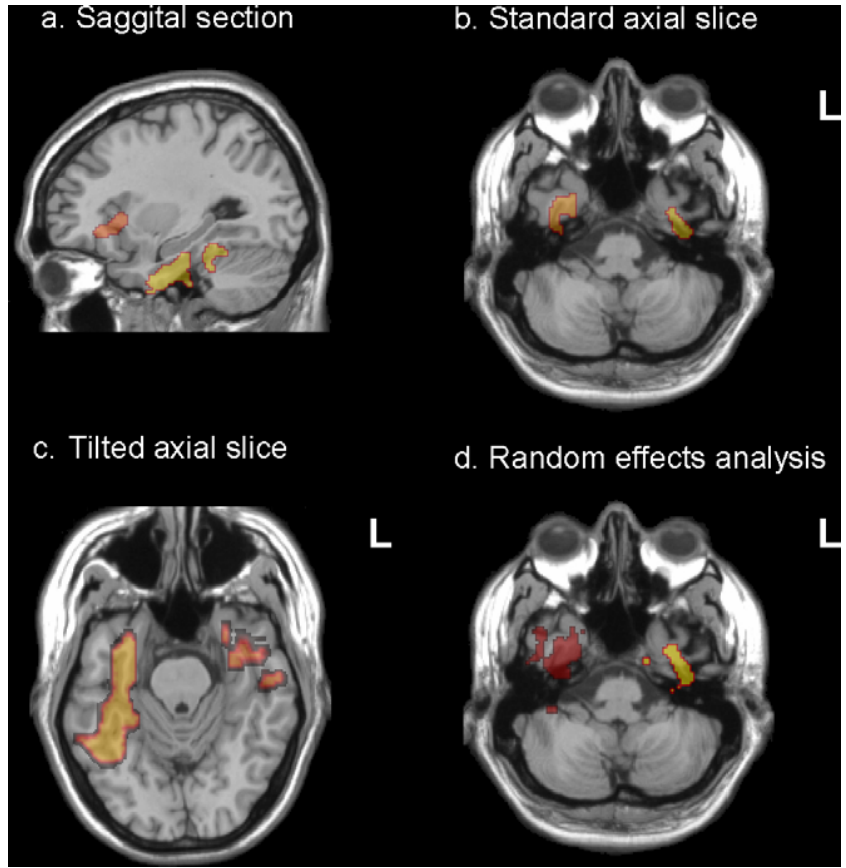

Fig. 1. Sections slices summarising significantly activated temporal lobe clusters arising from the comparison of semantic over control tasks.

Footnote: Results from a fixed effect analysis in which clusters are determined by $Z>3$ and corrected at $p=0.05$ (Worsley et al., 1992). (a) A left hemisphere sagittal section showing greater activation in fusiform and prefrontal regions; (b) An axial slice (in standard AC-PC orientation) showing greater activation in bilateral, anterior fusiform gyri extending to pole; (c) An axial slice (tilted at $25^{\circ}$ to AC-PC) showing activation along the length of the fusiform bilaterally but more extensively in the left hemisphere; and (d) An axial slice showing the same activated clusters after random-effects analysis with a cluster threshold of $Z>2$ and corrected at $p=0.05$.

cant potential benefits in these problematic regions. In addition, unlike some other corrective measures such as special shimming, this process retains whole-brain coverage.

A gradient reversal technique was used to correct geometrical distortion in the phase-encoding direction (Chang \& Fitzpatrick, 1992; Embleton et al., in press; Morgan et al., 2004). During a pre-scan volume, two images of each slice were acquired that were identical, except that the phase-encoding gradient was reversed. These images had equal geometrical distortion but in opposing directions along the phase-encoding gradient. Thus by integrating the signal of these images, the correct location can be determined (Chang \& Fitzpatrick, 1992; Embleton et al., in press; Morgan et al., 2004). This process generates a correction map of pixel displacement. The rest of the volumes were acquired in one phase-encoding direction. Each volume was registered to the original distorted mean pre-scan volume using a six-degrees of freedom translation and rotation algorithm (FLIRT, FSL, Oxford). The map of pixel displacement was then used to correct the distorted data. An in-house algorithm devised by Embleton et al. (in press) was used to register image pairs to sub-voxel accuracy in the frequency encoding direction. This resulted in a distortion-corrected dataset of 160 volumes maintaining the original temporal spacing and TR of $3200 \mathrm{~ms}$. In addition, the phase-encoding direction was set from left to right, instead of anterior to posterior for two reasons. First, the number of phase-encoding lines is reduced due to the rectangle shape of the brain. Secondly, eye movement causes distortion and by using this acquisition method only a few slices will include eye movement.

\subsection{FMRI data analyses}

Analysis was carried out using FEAT version 5.63 (FMRI Expert Analysis Tool), which is part of the FSL analysis package (FMRIB's Software Library, www.fmrib.ox.ac.uk/fsl). Single-subject analyses included the following prestatistics processing stages: motion correction using MCFLIRT (Jenkinson, Bannister, Brady, \& Smith, 2002); slice-timing correction using Fourier-space time-series phase-shifting; non-brain removal using BET (Smith, 2002); spatial smoothing using a Gaussian kernel of $8 \mathrm{~mm}$ FWHM; mean-based intensity normalisation of all volumes by the same factor; highpass temporal filtering (Gaussian-weighted least-squares straight line fitting, with sigma $=64.0 \mathrm{~s}$ ). Time-series statistical analysis was carried out using FILM with local autocorrelation correction (Woolrich, Ripley, Brady, \& Smith, 2001). Registration to high resolution and/or standard images was carried out using FLIRT (Jenkinson et al., 2002; Jenkinson \& Smith, 2001). 
Following Devlin et al. (2000), multi-subject analysis was carried out using a fixed effects model, by forcing the random-effects variance to zero in FLAME (FMRIB's local analysis of mixed effects: Beckmann, Jenkinson, \& Smith, 2003; Woolrich, Behrens, Beckmann, Jenkinson, \& Smith, 2004).Z(Gaussianised T/F) statistic images were thresholded using clusters determined by $Z>3.0$ and a (corrected) cluster significance threshold of $P<0.05$ (Worsley, Evans, Marrett, \& Neelin, 1992). The same analysis was repeated with clusters determined by $Z>2.3$. Furthermore, analyses were performed on the uncorrected data with clusters determined by $Z>1.7$. In addition, we included a random effect analysis to ensure activation was still present after accounting for the variation between subjects. Clusters were determined by $Z>2.0$. Functional images were overlaid on the MNI brain template using MRIcro (www.mricro.com) to investigate anatomical position of the activated clusters.

\section{Results}

The contrast of the semantic task against the control condition yielded five extensive regions of activation when the cluster was determined by fixed effect analyses and a $Z>3.0$. These regions are shown in Figs. 1 and 2c. Peak activations are listed in Table 1. The bilateral temporal cluster defined by a cluster threshold of $Z>3.0$ extends anterior to a $y$-value of 0 . The extent of these clusters can be described as follows. Anteriorly, the cluster includes the entorhinal cortex, perirhinal cortext and the posterior part of the temporal pole as defined by Insausti et al. (1998). This anterior part spreads more laterally in the right hemisphere (an $x$-range of 30-58 compared to -27 to -32 in the left hemisphere). When the cluster threshold was lowered to $Z>2.3$, the activation extended all the way to the anterior parts of the temporal poles (TP) as defined by Insausti et al. (1998); coordinates $(-32,21,-40)$ and $(-23,12,-40)$. This is presented in Fig. 2d. In the posterior part of the same cluster $(Z>3.0)$, the left temporal cluster is restricted to the fusiform gyrus until a $y$-value of -28 , but then spreads more laterally to the inferior temporal lobe extending posteriorly until a $y$-value of -62 . The cluster in the right temporal lobe remains relatively anterior extending to a $y$-value of -28 , covering the fusiform gyrus and inferior temporal lobe. Local maxima, peak activations of the left and right temporal clusters are listed in Tables 2 and 3.

In addition, the inferior frontal gyrus including the pars triangularis was activated bilaterally and the clusters extended to the middle frontal gyrus. In the left hemisphere, frontal activation included Broca's area (BA 45). Because we used the same tasks as

\section{(a) PET vs. fMRI comparison from Devlin et al. (2000)}

$\mathbf{L}$
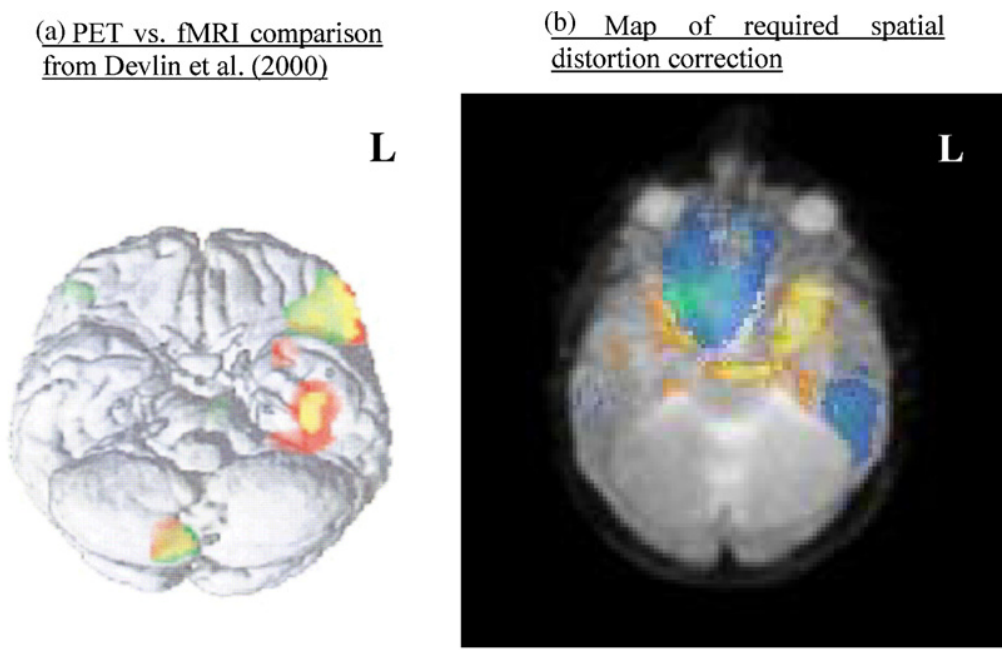

(c) Results from distortioncorrected fMRI at $\mathrm{z}>3$

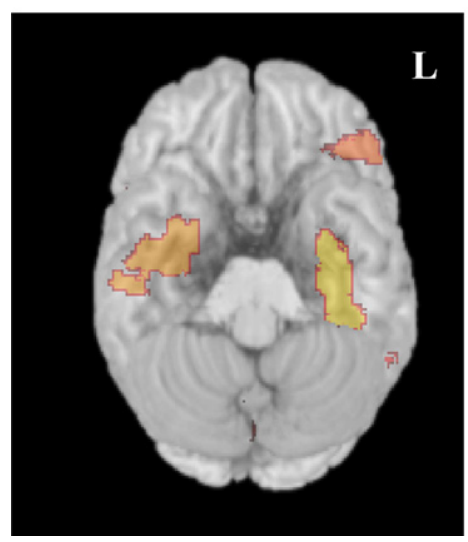

(d) Results from distortioncorrected fMRI at $\mathrm{z}>2.3$

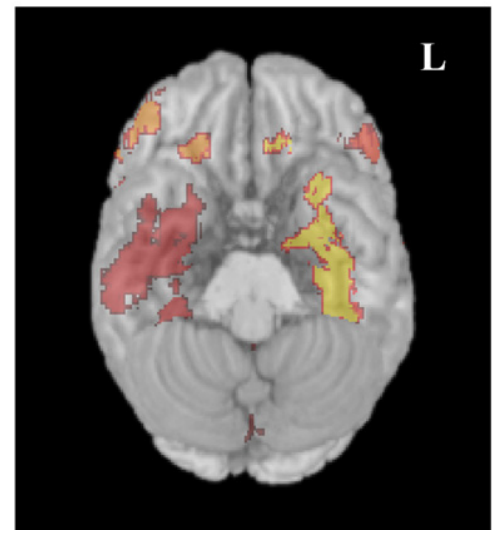

Fig. 2. Direct comparison of rendered images (ventral surface) from Devlin et al. (2000), required spatial distortion correction and resultant functional activations from the current study. (a) PET vs. fMRI comparison from Devlin et al. (2000). (b) Map of required spatial distortion correction. (c) Results from distortion-corrected fMRI at $Z>3$. (d) Results from distortion-corrected fMRI at $Z>2.3$.

Footnote: (a) Colour code as per original report: red denotes PET-only activation, green $=$ fMRI-only activation, yellow $=$ activation found in both imaging modalities (reproduced with permission from author). (b) Individual example of a corrected SE EPI data set ( $K_{\mathrm{L}}$ phase encode) showing the ventral surface; coloured overlays show the degree of spatial remapping required to correct the original distorted image. Yellow denotes that voxels were shifted right to left (maximum 5.85 voxels) and blue from left to right (maximum 12.23 voxels). As expected, minimal distortion correct is required throughout the brain except for two regions: (i) orbital frontal (middle blue region in the figure), and (ii) inferior and anterior temporal lobes bilaterally. Overlapping regions were activated in the PET study of Devlin et al. (2000) - see Fig. 2a - and is resolved in SE EPI fMRI once the distortion correction is applied (Fig. 1c). (c and d) Results from the current study, based on distortion-corrected SE EPI fMRI at 3T. Clusters are determined by $Z>3$ (c) and $Z>2.3$ (d) and corrected at $p=0.05$ (Worsley et al., 1992). Bilateral fusiform activation was found extending to temporal pole - consistent with semantic dementia. (For interpretation of the references to color in this figure legend, the reader is referred to the web version of the article.) 
Table 1

Activated clusters revealed by the contrast of semantic over letter (control) task.

\begin{tabular}{|c|c|c|c|c|c|}
\hline \multirow[t]{2}{*}{ Brain region (COG) } & \multirow[t]{2}{*}{ Voxels } & \multirow[t]{2}{*}{ Max. $Z$ value } & \multicolumn{3}{|c|}{ MNI coordinates } \\
\hline & & & $\overline{\text { Mean } x}$ & Mean $y$ & Mean $z$ \\
\hline L. Fusiform gyrus & 4306 & 6.13 & -44 & -58 & -12 \\
\hline R. Inferior frontal gyrus & 3435 & 5.76 & 36 & 22 & 0 \\
\hline L. Inferior frontal gyrus & 2721 & 5.58 & -38 & 4 & 32 \\
\hline R. Fusiform gyrus & 2586 & 4.58 & 40 & -8 & -38 \\
\hline Cerebellum & 1146 & 5.12 & 6 & -82 & -20 \\
\hline
\end{tabular}

MNI coordinates of the centre of gravity (COG) of each cluster, determined by $Z>3$ and a (corrected) cluster significance threshold of $P=0.05$.

Table 2

Local maxima in the right temporal lobe cluster revealed by the contrast of semantic over letter (control) task.

\begin{tabular}{|c|c|c|c|c|c|}
\hline \multirow[t]{2}{*}{ Brain region (COG) } & \multirow[t]{2}{*}{$\mathrm{BA}(\mathrm{COG})$} & \multirow[t]{2}{*}{$Z$ value } & \multicolumn{3}{|c|}{ MNI coordinates } \\
\hline & & & Mean $x$ & Mean $y$ & Mean $z$ \\
\hline Fusiform gyrus & 20 & 4.58 & 40 & -8 & -38 \\
\hline Fusiform gyrus & 20 & 4.16 & 34 & -12 & -40 \\
\hline Fusiform gyrus & 20 & 4.09 & 58 & -20 & -26 \\
\hline Fusiform gyrus & 20 & 4.03 & 52 & -8 & -40 \\
\hline Anterior temporal lobe & 38 & 4.01 & 28 & 2 & -42 \\
\hline Cerebellum & & 3.75 & 38 & -34 & -38 \\
\hline
\end{tabular}

MNI coordinates of the centre of gravity (COG) of each cluster, determined by $Z>3$ and a (corrected) cluster significance threshold of $P=0.05$.

Devlin et al. (2000), the current study did not control for executive demands between the semantic and the control task and, therefore, we cannot verify whether this frontal activation can be attributed to general executive processes or to specific semantic control processes. Previous literature has associated frontal areas with both types of processing (Banich et al., 2000; Jefferies \& Lambon Ralph, 2006). Finally, a cluster in the cerebellum was activated.

In order to provide directly comparable data to Devlin et al. (2000), both the same task and analysis (fixed-effects) were used. To check for the generalisation of findings across participants, we also conducted a random-effects analysis. This resulted in the same activated clusters (see Fig. 1d). In contrast to the fMRI results reported by Devlin et al. (2000), our fMRI results show good comparisons with the PET images from their study - which, for ease, are reproduced in Fig. 2a [PET (red) and fMRI (green)]. We found the same areas activated but, in addition, the current study highlighted bilateral ATL activation as opposed to the unilateral activation in Devlin's et al. (2000) study. As noted in Section 1, the fact that we identified bilateral activation in this study is consistent with the bilateral ATL atrophy associated with semantic dementia and the effects of left or right temporal pole rTMS in normal participants (Davies et al., 2004; Lambon Ralph et al., 2009; Nestor et al., 2006).

It should be noted that the improved fMRI results obtained in this study, probably reflect a combination of three factors: advances in MR engineering (e.g., SENSE head coils), the use of SE EPI and the distortion correction. This study was not designed to measure the efficacy of each factor, independently, as it was focussed primarily on an applied research question (which regions of the ATL contribute to semantic memory). We can be fairly certain that the distortion correction made a significant, independent contribution for two reasons: first, when we repeated the same analyses but on the uncorrected data (i.e., SE EPI data acquired with SENSE head coils), the ATL activations were not present. Secondly, the amount of spatial correction varies systematically across the brain. Fig. $2 b$ shows an individual EPI volume after the spatial correction has been applied. The overlay denotes the amount of spatial correction required to bring each voxel back to its correct position. This shows very clearly that, in addition to adjustment for orbitofrontal regions, considerable correction is required for much of the ventral surface of the temporal lobes (and to a lesser extent the polar and lateral aspects of the ATL too). The differences between GE and SE EPI can be seen in the within-participant comparison (see
Fig. 3). As noted in Section 1, where EPI signal is present (displayed in the greyscale, coronal sections) then the signal-to-noise (coloured coronal sections: calculated as per Murphy, Bodurkab, \& Bandettinia, 2007) is generally higher in GE than SE. However, in some regions there is clear absence of signal in the GE EPI scans which is retained in SE EPI. As well as orbitofrontal areas, these problematic regions include the anterior, inferior temporal lobe and inferior polar regions. Given this is exactly the same region that was activated by this study and other PET-based investigations (Crinion, Lambon Ralph, Warburton, Howard, \& Wise, 2003; Scott, Blank, Rosen, \& Wise, 2000; Wise, 2003), then it is not so surprising that the majority of GE EPI fMRI studies fail to observe inferolateral, ATL activations.

On a purely practical final note, we would suggest that this recipe of imaging factors (SE EPI, SENSE or its equivalents, and spatial correction) is a good one if researchers are keen to probe the functioning of the ATL or other areas affected by susceptibility artefacts, whilst also maintaining whole-brain coverage. In any event, it should be remembered that it is difficult to conclude much about

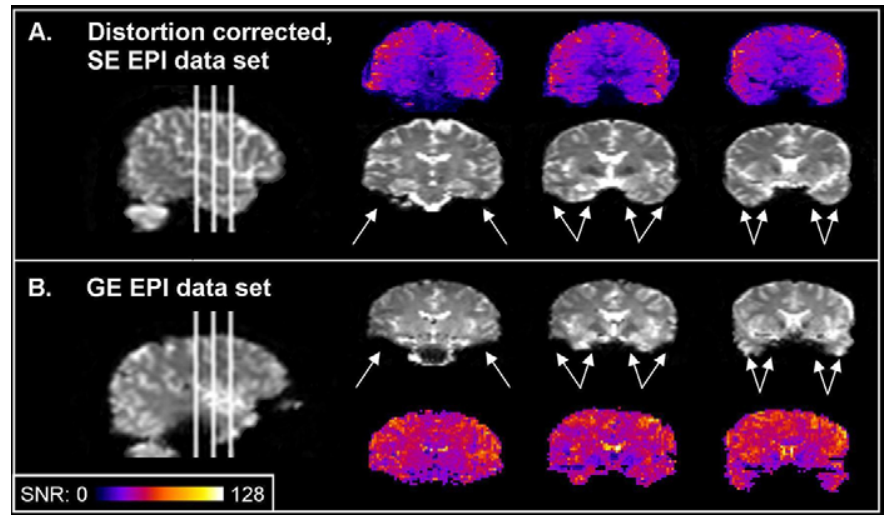

Fig. 3. Within-participant comparison of gradient-echo vs. spin-echo EPI. Footnote: Comparative spin-echo (panel A) vs. gradient-echo EPI images (panel B) from the same participant. Both raw EPI and SNR maps (Murphy et al., 2007) are displayed. Two key differences can be observed. Where EPI signal is present, then SNR tends to be higher for GE than SE data. In the GE scans, however, there are regions of signal drop out (marked by white arrows) - in orbitofrontal, inferior-anterior and polar temporal lobe regions. Signal is present, however, in the same regions for the SE-EPI. 
Table 3

Local maxima in left temporal lobe cluster revealed by the contrast of semantic over letter (control) task.

\begin{tabular}{|c|c|c|c|c|c|}
\hline \multirow[t]{2}{*}{ Brain region (COG) } & \multirow[t]{2}{*}{$\mathrm{BA}(\mathrm{COG})$} & \multirow[t]{2}{*}{$Z$ value } & \multicolumn{3}{|c|}{ MNI coordinates } \\
\hline & & & Mean $x$ & Mean $y$ & Mean $z$ \\
\hline Inferior temporal gyrus & 20 & 6.13 & -44 & -58 & -12 \\
\hline Inferior temporal gyrus & 20 & 6.09 & -42 & -50 & -14 \\
\hline Fusiform gyrus & 20 & 5.78 & -36 & -14 & -40 \\
\hline Fusiform gyrus & 20 & 5.3 & -44 & -40 & -14 \\
\hline Fusiform gyrus & 20 & 4.95 & -36 & -28 & -22 \\
\hline Fusiform gyrus & 20 & 4.83 & -34 & -20 & -28 \\
\hline
\end{tabular}

MNI coordinates of the centre of gravity (COG) of each cluster, determined by $Z>3$ and a (corrected) cluster significance threshold of $P=0.05$.

the inferior ATL if only standard GE EPI is used. On the flip side, given the better sensitivity and temporal resolution of GE over SE EPI, the former is favourable for fMRI studies when the areas of interests are not prone to the susceptibility artefact. These limitations of SE EPI may be more problematic in event-related designs.

\section{Discussion}

Patients with semantic dementia (SD) have a specific impairment of semantic memory following bilateral atrophy of the anterior temporal lobes (ATL), suggesting that areas within this region form a critical substrate for semantic memory (Lambon Ralph \& Patterson, 2008; Patterson et al., 2007). In addition, PET neuroimaging and rTMS studies of healthy subjects show the importance of the ATL in semantic processing (Lambon Ralph et al., 2008; Pobric et al., 2007; Vandenberghe et al., 1996; Wise, 2003). In contrast, previous fMRI studies on semantic memory have largely failed to observe activation within the ATL (Visser et al., 2010) and, perhaps because of the predominance of fMRI in cognitive neuroscience, the role of the ATL in semantic cognition has tended to be overlooked. Indeed, several recent reviews of the neural basis of semantic memory have not pointed to any involvement of this region (Catani \& Ffytche, 2005; Mesulam, 1998). We suggest that this disparity across neuroscience methods reflects a series of factors that generate a relative absence of neuroimaging evidence with regard to the ATL. Visser et al. (2010) found that the likelihood of finding evidence of ATL activations in the literature reflected the size of the field-of-view used, the nature of the baseline task and whether the imaging technique suffered from susceptibility artefacts. By adopting a whole-brain, SE EPI fMRI technique with a spatial correction and parallel receiver coils (Embleton et al., in press), we were able to demonstrate that the inferior, anterior temporal lobes are bilaterally activated by a rapid semantic categorisation task (previously used by Devlin et al., 2000). As such, these results are in line with the investigations of semantic dementia and recent rTMS studies. It should be noted that we found activation in this inferior ATL region despite the fact that participants were making category-membership decisions to basic level concepts. This would suggest that this region processes all levels of concept and not just subordinate exemplars or specific entities (Martin, 2007; Martin et al., 1996; Tranel et al., 1997; Tyler et al., 2004).

The observation of anterior, inferior ATL activation is important for two reasons: (a) this study provides convergence results across neuropsychology, rTMS and neuroimaging, implicating the importance of the inferior aspects of ATL regions in semantic cognition; and (b) it licenses the use of this form of distortion-corrected fMRI in future studies. These investigations will be able to explore which aspects of the ATL are particularly important in semantic cognition and whether these vary according to important factors such as category/domain, modality of input, specificity of processing and so on. We should note that distortion correction can be achieved using methods other than the reversed gradient approach used in this study. For example, corrections based on field mapping methods (Andersson, Hutton, Ashburner, Turner, \& Friston, 2001; Hutton et al., 2002; Jezzard et al., 2001; Reber, Wong, Buxton, \& Frank, 1998; Yeo, Fessler, \& Kim, 2008) can successfully be combined with SE-EPI for fMRI. It remains for future work to assess the relative benefits of different distortion correction methods. In any event, the purpose and conclusion of the present study is unchanged: with correction, fMRI studies of semantic behaviour align with the rest of the neuroscience literature and implicate the anterior temporal lobes in semantic cognition.

By adopting the same behavioural paradigm, procedure and analysis, we were able to compare our results directly with those reported previously by Devlin et al. (2000). As expected, our findings from this study are similar to the PET neuroimaging results from Devlin et al. (2000). Both studies revealed a large cluster of activation in the left hemisphere extending from the inferior ATL to the middle temporal cortex, the inferior and middle frontal regions and, in addition, a cluster in the cerebellum. Our study also revealed involvement of right anterior, inferolateral temporal regions. This is of particular interest because semantic dementia always arises in the context of bilateral ATL atrophy/hypometabolism and recent direct comparisons of left vs. right rTMS have found equivalent effects on semantic decision times (Lambon Ralph et al., 2009). Taken together, these results suggest that the contribution made by the ATL - especially its inferior aspects - to semantic memory is reflective of a bilateral, neurally coupled system. In another rTMS study (Pobric et al., in press), we have replicated the previous result (slowed semantic decisions after left or right ATL stimulation) and have found that it holds whether semantic decisions are made to either pictured or verbal labelled concepts (timed semantic association decisions to pictures or written names). This result seems to rule out a strong division of labour across the two ATL regions in terms of verbal vs. nonverbal semantic memory. Instead, it seems likely that bilateral representation of amodal semantic concepts has beneficial value in that the representations become more robust to damage or neural disruption.

Nonetheless, the right ATL cluster revealed in this study was somewhat weaker than the cluster in the left ATL, perhaps reflecting the importance of language processing in these particular semantic tasks. This could result from differential connectivity from the bilateral amodal semantic system to left dominant language centres (Lambon Ralph, McClelland, Patterson, Galton, \& Hodges, 2001). Consistent with this, a recent combined re-analysis of 18 PET studies involving object naming tasks found bilateral ATL activation, which was stronger on the left (Price, Devlin, Moore, Morton, \& Laird, 2005). Therefore, the absence of activation in the right ATL in Devlin et al.'s (2000) study might reflect the fact that their method was not sensitive enough to detect the weaker activation in the right ATL. Generally fMRI is thought to be more sensitive to activation than PET, because more data are acquired over the same time (Mechelli, Friston, \& Price, 2000; Owen \& Coleman, 2007; Owen, Epstein, \& Johnsrude, 2001). Furthermore, differences in spatial resolution, smoothing kernels and signal characteristics might con- 
tribute to the discrepancies between fMRI and PET results (Price, Veltman, Ashburner, Josephs, \& Friston, 1999).

This study also allows us to begin to examine more specific neuroanatomical questions. Volumetric studies have reported bilateral, anterior temporal atrophy in SD patients (Davies et al., 2004; Galton et al., 2001; Gorno-Tempini et al., 2004; Mummery et al., 2000; Nestor et al., 2006). Although the atrophy in SD extends across the entire anterior temporal lobe region, it tends to be particularly pronounced in the inferolateral aspects (atrophy being the greatest in the perirhinal, fusiform and ITG regions: Davies et al., 2004). This is intriguing because (a) these are the same areas that were maximally activated in this fMRI study (and other PET studies) and (b) are some of the regions that suffer the most from distortion arising from magnetic field inhomogeneities (see Fig. $2 b$ and Fig. 3). The fact that these three findings point to the same regions, not only indicates the important of these areas in semantic cognition but also might be the root of the previous disparity between fMRI and patient-studies of semantic memory. Future studies will be able to test whether this region is implicated in all semantic tasks (e.g., irrespective of modality of input) and also its relationship to other ATL regions that have been highlighted in other PET studies (e.g., the anterolateral STS: Scott et al., 2000).

Given that many theories of semantic cognition do not include or mention ATL regions at all (see above), it is useful to consider what function the keys areas within this region may bring to semantic cognition. It is clear from this and many other patient and neuroimaging studies that semantic cognition (semantically driven behaviour) is supported by a network of brain regions. Broadly-speaking these can be split into two types: informationbearing regions and control/regulatory areas. Following models of cognitive control (Garavan, Ross, Li, \& Stein, 2000; Peers et al., 2005), there is now fMRI, rTMS and patient evidence to suggest that prefrontal and temporoparietal regions help to shape or in some way regulate which aspects of meaning are relevant to the language or nonverbal activity in hand (Devlin, Matthews, \& Rushworth, 2003; Jefferies, Baker, Doran, \& Lambon Ralph, 2007; Jefferies \& Lambon Ralph, 2006; Noonan, Jefferies, Corbett, \& Lambon Ralph, 2010; Thompson-Schill et al., 1997). These regions are especially important, therefore, when complex semantic judgements are needed, when there is strong intrinsic competition between alternative meanings and in everyday verbal and nonverbal activities that require some but not all aspects of the meaning.

Many models of semantic memory (that is of semantic information rather than semantic control) emphasise that concepts may be formed by linking together different types of modalityspecific information (e.g., what things look, smell, taste like and the words we use to describe them) arising in different association areas (an idea that stems back to Wernicke and Meynert: Eggert, 1977; Martin, 2007). Rogers et al. (2004) have suggested that the ATL works as a representational hub, connecting modalityspecific information to form amodal semantic representations. Such a hub is required when performing complex generalisations, based on central semantic relationships rather than superficial similarities found in one particular domain (e.g., being able to recognize a Chihuahua as dog even though it does not look like a dog) (Lambon Ralph \& Patterson, 2008; Lambon Ralph, Sage, Jones, \& Mayberry, 2010). These computational challenges are hard, if not impossible, to overcome by directly connecting different types of modality-specific information (Rogers et al., 2004). Implemented computational models indicate, however, that successful conceptualisation can follow if modality-specific information is combined and translated via an additional, single set of amodal units (Rogers et al., 2004). Our working hypothesis, therefore, is that ATL regions (via their wide spread neocortical connectivity: Gloor, 1997) provide the neural basis for this central and critical aspect of semantic memory (Lambon Ralph \& Patterson, 2008; Lambon Ralph et al., 2010).

\section{Acknowledgements}

This study was supported by an MRC programme grant (G0501632) and MRC Pathfinder Grant (G0300952).

\section{References}

Andersson, J. L. R., Hutton, C., Ashburner, J., Turner, R., \& Friston, K. (2001). Modeling geometric deformations in EPI time series. Neurolmage, 13(5), 903-919.

Banich, M. T., Milham, M. P., Atchley, R., Cohen, N. J., Webb, A., Wszalek, T., et al. (2000). fMRI studies of stroop tasks reveal unique roles of anterior and posterior brain systems in attentional selection. Journal of Cognitive Neuroscience, 12(6), 988-1000

Beckmann, C. F., Jenkinson, M., \& Smith, S. M. (2003). General multilevel linear modeling for group analysis in fmri. NeuroImage, 20(2), 1052-1063.

Binder, J. R., Desai, R. H., Graves, W. W., \& Conant, L. L. (2009). Where is the semantic system? A critical review and meta-analysis of 120 functional neuroimaging studies. Cerebral Cortex, 55.

Binder, J. R., Frost, J. A., Hammeke, T. A., Bellgowan, P. S. F., Rao, S. M., \& Cox, R. W. (1999). Conceptual processing during the conscious resting state: A functional MRI study. Journal of Cognitive Neuroscience, 11(1), 80-93.

Brambati, S. M., Rankin, K. P., Narvid, J., Seeley, W. W., Dean, D., Rosen, H. J., et al. (2009). Atrophy progression in semantic dementia with asymmetric temporal involvement: A tensor-based morphometry study. Neurobiology of Aging, 30(1), 103-111.

Canessa, N., Borgo, F., Cappa, S. F., Perani, D., Falini, A., Buccino, G., et al. (2007) The different neural correlates of action and functional knowledge in semantic memory: An fMRI study. Cerebral Cortex, 18(4), 740-751.

Catani, M., \& Ffytche, D. H. (2005). The rises and falls of disconnection syndromes. Brain, 128(10), 2224-2239.

Chang, H., \& Fitzpatrick, J. M. (1992). A technique for accurate magnetic-resonanceimaging in the presence of field inhomogeneities. IEEE Transactions on Medical Imaging, 11(3), 319-329.

Chao, L. L., \& Martin, A. (2000). Representation of manipulable man-made objects in the dorsal stream. NeuroImage, 12(4), 478-484.

Crinion, J. T., Lambon Ralph, M. A., Warburton, E. A., Howard, D., \& Wise, R. J. S. (2003). Temporal lobe regions engaged during normal speech comprehension. Brain, 126, 1193-1201.

Damasio, A \& Damasio, H. (1994). Cortical systems for retrieval of concrete knowledge: The convergence zone framework. In C. Koch, \& J. Davis (Eds.), Large-scale neuronal theories of the brain (pp. 61-74). Cambridge, MA: The MIT Press.

Davies, R. R., Graham, K. S., Xuereb, J. H., Williams, G. B., \& Hodges, J. R. (2004). The human perirhinal cortex and semantic memory. European Journal of Neuroscience, 20(9), 2441-2446.

Deichmann, R., Josephs, O., Hutton, C., Corfield, D. R., \& Turner, R. (2002). Compensation of susceptibility-induced BOLD sensitivity losses in echo-planar fMRI imaging. Neurolmage, 15(1), 120-135.

Demb, J. B., Desmond, J. E., Wagner, A. D., Vaidya, C. J., Glover, G. H., \& Gabrieli, J. D. E. (1995). Semantic encoding and retrieval in the left inferior prefrontal cortex: A functional MRI study of task-difficulty and process specificity. Journal of Neuroscience, 15(9), 5870-5878.

Devlin, J. T., Matthews, P. M., \& Rushworth, M. F. S. (2003). Semantic processing in the left inferior prefrontal cortex: A combined functional magnetic resonance imaging and transcranial magnetic stimulation study. Journal of Cognitive Neuroscience, 15(1), 71-84.

Devlin, J. T., Russell, R. P., Davis, M. H., Price, C. J., Wilson, J., Moss, H. E., et al. (2000). Susceptibility-induced loss of signal: Comparing PET and fMRI on a semantic task. NeuroImage, 11(6), 589-600.

Eggert, G. H. (1977). Wernicke's work on aphasia; a source-book and review. The Hague: Mouton.

Embleton, K., Haroon, H., Lambon Ralph, M. A., Morris, D., \& Parker, G. J. M. Distortion correction for diffusion weighted MRI tractogrophy and fMRI in the temporal lobes. Human Brain Mapping, in press, doi:10.1002/hbm.20959.

Galton, C. J., Patterson, K., Graham, K., Lambon Ralph, M. A., Williams, G., Antoun, N., et al. (2001). Differing patterns of temporal atrophy in Alzheimer's disease and semantic dementia. Neurology, 57(2), 216-225.

Garavan, H., Ross, T. J., Li, S. J., \& Stein, E. A. (2000). A parametric manipulation of central executive functioning. Cerebral Cortex, 10(6), 585-592.

Gloor, P. (1997). The temporal lobe and the limbic system. Oxford: Oxford University Press.

Gorno-Tempini, M. L., Dronkers, N. F., Rankin, K. P., Ogar, J. M., Phengrasamy, L., Rosen, H. J., et al. (2004). Cognition and anatomy in three variants of primary progressive aphasia. Annals of Neurology, 55(3), 335-346.

Hickok, G., \& Poeppel, D. (2007). The cortical organization of speech processing. Nature Reviews Neuroscience, 8(5), 393-402.

Hodges, J. R., \& Patterson, K. (1995). Is semantic memory consistently impaired early in the course of Alzheimer's disease? Neuroanatomical and diagnostic implications. Neuropsychologia, 33, 441-459.

Hodges, J. R., Patterson, K., Oxbury, S., \& Funnell, E. (1992). Semantic dementia: Progressive fluent aphasia with temporal-lobe atrophy. Brain, 115, 1783-1806. 
Hutton, C., Bork, A, Josephs, O., Deichmann, R., Ashburner, J., \& Turner, R. (2002) Image distortion correction in fMRI: A quantitative evaluation. Neurolmage $16(1), 217-240$

Insausti, R., Juottonen, K., Soininen, H., Insausti, A. M., Partanen, K., Vainio, P., et al. (1998). MR volumetric analysis of the human entorhinal, perirhinal, and temporopolar cortices. American Journal of Neuroradiology, 19(4), 659-671.

Jefferies, E., Baker, S. S., Doran, M., \& Lambon Ralph, M. A. (2007). Refractory effects in stroke aphasia: A consequence of poor semantic control. Neuropsychologia 45, 1065-1079.

Jefferies, E., \& Lambon Ralph, M. A. (2006). Semantic impairment in stroke aphasia versus semantic dementia: A case-series comparison. Brain, 129, 2132-2147.

Jenkinson, M., Bannister, P., Brady, M., \& Smith, S. (2002). Improved optimization for the robust and accurate linear registration and motion correction of brain images. Neurolmage, 17(2), 825-841.

Jenkinson, M., \& Smith, S. (2001). A global optimisation method for robust affine registration of brain images. Medical Image Analysis, 5(2), 143-156.

Jezzard, P., Matthews, P. M., \& Smith, S. M. (2001). Functional MRI: An introduction to methods. Oxford: Oxford University press.

Jones, R. A., Brookes, J. A. \& Moonen, C. T. W. (2001). Ultra-fast fMRI. In P. Jezzard, P. M. Matthews, \& S. M. Smith (Eds.), Functional MRI: An introduction to methods (pp. 93-109). New York: Oxford University Press.

Lambon Ralph, M. A., Lowe, C., \& Rogers, T. T. (2007). Neural basis of category-specific semantic deficits for living things: Evidence from semantic dementia, hsve and a neural network model. Brain, 130(4), 1127-1137.

Lambon Ralph, M. A., McClelland, J. L., Patterson, K., Galton, C. J., \& Hodges, J. R. (2001). No right to speak? The relationship between object naming and semantic impairment: Neuropsychological abstract evidence and a computational model. Journal of Cognitive Neuroscience, 13(3), 341-356.

Lambon Ralph, M. A., \& Patterson, K. (2008). Generalization and differentiation in semantic memory: Insights from semantic dementia. Annals of the New York Academy of Sciences, 1124, 61-76.

Lambon Ralph, M. A., Pobric, G., \& Jefferies, E. (2008). Conceptual knowledge is underpinned by the temporal pole bilaterally: Convergent data from rTMS. Cerebral Cortex, Advance access published online on August 4, 2008 , doi:10.1093/cercor/bhn131.

Lambon Ralph, M. A., Pobric, G., \& Jefferies, E. (2009). Conceptual knowledge is underpinned by the temporal pole bilaterally: Convergent evidence from rTMS Cerebral Cortex, 19(4), 832-838.

Lambon Ralph, M. A., Sage, K., Jones, R.W., \& Mayberry, E. J. (2010). Coherent concepts are computed in the anterior temporal lobes. PNAS, 107, 2717-2722.

Li, Y., Xu, N., Fitzpatrick, J. M., Morgan, V. L., Pickens, D. R., \& Dawant, B. M. (2007). Accounting for signal loss due to dephasing in the correction of distortions in gradient-echo EPI via nonrigid registration. IEEE Transactions on Medical Imaging 26(12), 1698-1707.

Marinkovic, K., Dhond, R. P., Dale, A. M., Glessner, M., Carr, V., \& Halgren, E. (2003). Spatiotemporal dynamics of modality-specific and supramodal word processing. Neuron, 38(3), 487-497.

Martin, A. (2007). The representation of object concepts in the brain. Annual Review of Psychology, 58, 25-45.

Martin, A., Wiggs, C. L., Ungerleider, L. G., \& Haxby, J. V. (1996). Neural correlates of category-specific knowledge. Nature, 379(6566), 649-652.

McKiernan, K. A., D’Angelo, B. R., Kaufman, J. N., \& Binder, J. R. (2006). Interrupting the "Stream of consciousness": An fMRI investigation. Neurolmage, 29(4), 1185-1191.

Mechelli, A., Friston, K. J., \& Price, C. J. (2000). The effects of presentation rate during word and pseudoword reading: A comparison of PET and fMRI. Journal of Cognitive Neuroscience, 12, 145-156.

Mechelli, A., Sartori, G., Orlandi, P., \& Price, C. J. (2006). Semantic relevance explains category effects in medial fusiform gyri. Neurolmage, 30(3), 992-1002.

Mesulam, M. M. (1998). From sensation to cognition. Brain, 121, 1013-1052.

Morgan, P.S., Bowtell, R. W., McIntyre, D. J. O., \& Worthington, B. S. (2004). Correction of spatial distortion in EPI due to inhomogeneous static magnetic fields using the reversed gradient method. Journal of Magnetic Resonance Imaging, 19(4), 499-507.

Mummery, C. J., Patterson, K., Price, C. J., Ashburner, J., Frackowiak, R. S. J., \& Hodges, J. R. (2000). A voxel-based morphometry study of semantic dementia: Relationship between temporal lobe atrophy and semantic memory. Annals of Neurology, 47(1), 36-45.

Murphy, K., Bodurkab, J., \& Bandettinia, P. A. (2007). How long to scan? The relationship between fMRI temporal signal to noise ratio and necessary scan duration. NeuroImage, 34(2), 565-574.

Nestor, P. J., Fryer, T. D., \& Hodges, J. R. (2006). Declarative memory impairments in Alzheimer's disease and semantic dementia. Neurolmage, 30(3), 1010-1020.

Noonan, K. A., Jefferies, E., Corbett, F., \& Lambon Ralph, M. A. Elucidating the nature of deregulated semantic cognition in semantic aphasia: Evidence for the roles of prefrontal and temporoparietal cortices. Journal of Cognitive Neuroscience, 2010 , in press.

Noppeney, U., Patterson, K., Tyler, L. K., Moss, H., Stamatakis, E. A., Bright, P., et al. (2007). Temporal lobe lesions and semantic impairment: A comparison of herpes simplex virus encephalitis and semantic dementia. Brain, 130, 1138-1147.
Owen, A. M. \& Coleman, M. R. (2007). Functional MRI in disorders of consciousness: Advantages and limitations. Current Opinion in Neurology, 20(6), 632-637.

Owen, A. M., Epstein, R., \& Johnsrude, I. S. (2001). fMRI: Applications to cognitive neuroscience. In P. Jezzard, P. M. Mathews, \& S. M. Smith (Eds.), Functional magnetic resonance imaging: An introduction to methods (pp. 311-327). Oxford: Oxford University Press.

Patterson, K., Nestor, P. J., \& Rogers, T. T. (2007). Where do you know what you know? The representation of semantic knowledge in the human brain. Nature Reviews Neuroscience, 8(12), 976-987.

Peers, P. V., Ludwig, C. J. H., Rorden, C., Cusack, R., Bonfiglioli, C., Bundesen, C., et al. (2005). Attentional functions of parietal and frontal cortex. Cerebral Cortex 15(10), 1469-1484.

Pilgrim, L. K., Fadili, J., Fletcher, P., \& Tyler, L. K. (2002). Overcoming confounds of stimulus blocking: An event-related fMRI design of semantic processing. NeuroImage, 16(3), 713-723.

Pobric, G., Jefferies, E., \& Lambon Ralph, M. A. (2007). Anterior temporal lobes mediate semantic representation: Mimicking semantic dementia by using rTMS in normal participants. Proceedings of the National Academy of Sciences, 104(50) 20137-20141.

Pobric, G., Jefferies, E., \& Lambon Ralph, M. A. Amodal semantic representations depend on both left and right anterior temporal lobes: New rTMS evidence. Neuropsychologia, in press, doi:10.1016/j.neuropsychologia.2009.12.036.

Price, C. J., Devlin, J. T., Moore, C. J., Morton, C., \& Laird, A. R. (2005). Meta-analyses of object naming: Effect of baseline. Human Brain Mapping, 25(1), 70-82.

Price, C. J., Veltman, D. J., Ashburner, J., Josephs, O., \& Friston, K. J. (1999). The critical relationship between the timing of stimulus presentation and data acquisition in blocked designs with fMRI. NeuroImage, 10(1), 36-44.

Reber, P. J., Wong, E. C., Buxton, R. B., \& Frank, L. R. (1998). Correction of off resonancerelated distortion in echo-planar imaging using EPI-based field maps. Magnetic Resonance in Medicine, 39(2), 328-330.

Rogers, T. T., Lambon Ralph, M. A., Garrard, P., Bozeat, S., McClelland, J. L., Hodges, J. R., et al. (2004). Structure and deterioration of semantic memory: A neuropsychological and computational investigation. Psychological Review, 111(1), 205-235.

Schmithorst, V. J., Dardzinski, D. J., \& Holland, S. K. (2001). Simultaneous correction of ghost and geometric distortion artifacts in EPI using a multi-echo reference scan. IEEE Transactions on Medical Imaging, 20(6), 535-539.

Scott, S. K., Blank, C. C., Rosen, S., \& Wise, R. J. S. (2000). Identification of a pathway for intelligible speech in the left temporal lobe. Brain, 123(12), 2400-2406.

Smith, M. S. (2002). Fast robust automated brain extraction. Human Brain Mapping 17(3), 143-155.

Snowden, J. S., Griffiths, H. L., Neary, D., \& Mann, D. (1989). Semantic dementia: A form of circumscribed cerebral atrophy. Behavioural Neurology, 2, 167-182.

Thompson-Schill, S. L., D’Esposito, M., Aguirre, G. K., \& Farah, M. J. (1997). Role of left inferior prefrontal cortex in retrieval of semantic knowledge: A reevaluation. Proceedings of the National Academy of Sciences, 94(26), 14792-14797.

Tranel, D., Damasio, H., \& Damasio, A. R. (1997). A neural basis for the retrieval of conceptual knowledge. Neuropsychologia, 35(10), 1319-1327.

Tranel, D., Grabowski, T. J., Lyon, J., \& Damasio, H. (2005). Naming the same entities from visual or from auditory stimulation engages similar regions of left inferotemporal cortices. Journal of Cognitive Neuroscience, 17(8), 1293-1305.

Tyler, L. K., Stamatakis, E. A., Bright, P., Acres, K., Abdallah, S., Rodd, J. M., et al (2004). Processing objects at different levels of specificity. Journal of Cognitive Neuroscience, 16(3), 351-362.

Vandenberghe, R., Price, C., Wise, R., Josephs, O., \& Frackowiak, R. S. J. (1996). Functional anatomy of a common semantic system for words and pictures. Nature 383(6597), 254-256.

Visser, M., Jefferies, E \& Lambon Ralph, M. A (2010). Semantic processing in the anterior temporal lobes: A meta-analysis of the functional neuroimaging literature. Journal of Cognitive Neuroscience, 22, 1083-1094.

Weiskopf, N., Hutton, C., Josephs, O., \& Deichmann, R. (2006). Optimal EPI parameters for reduction of susceptibility-induced BOLD sensitivity losses: A whole-brain analysis at 3T and 1.5T. NeuroImage, 33(2), 493-504.

Wise, R. (2003). Language systems in normal and aphasic human subjects: Functional imaging studies and inferences from animal studies. British Medical Bulletin, 65, 95-119.

Woolrich, M. W., Behrens, T. E. J., Beckmann, C. F., Jenkinson, M., \& Smith, S. M. (2004) Multilevel linear modelling for fmri group analysis using bayesian inference. NeuroImage, 21(4), 1732-1747.

Woolrich, M. W., Ripley, B. D., Brady, M., \& Smith, S. M. (2001). Temporal autocorrelation in univariate linear modeling of fmri data. Neurolmage, 14(6), 1370-1386.

Worsley, K. J.. Evans, A. C., Marrett, S., \& Neelin, P. (1992). A three-dimensional statistical analysis for cbf activation studies in human brain. Journal of Cerebral Blood Flow and Metabolism, 12, 900-918.

Yeo, D. T. B., Fessler, J. A., \& Kim, B. (2008). Concurrent correction of geometric distortion and motion using the map-slice-to-volume method in echo-planar imaging. Magnetic Resonance Imaging, 26(5), 703-714. 